\title{
Políticas sobre violencias y abusos sexuales en las universidades catalanas
}

\author{
Policies on violence and sexual abuse in Catalan universities
}

SARA CAGLIERO

Universitat Rovira i Virgili

BARBARA BIGLIA

Universitat Rovira i Virgili

\section{Cómo citar/Citation}

Cagliero, S. y Biglia, B. (2019). Políticas sobre violencias y abusos sexuales en las universidades catalanas. Revista Española de Ciencia Política, 50, 141-170. Doi: https://doi.org/10.21308/recp.50.06

\section{Resumen}

Las universidades no son espacios libres de violencia, por lo que cabe preguntarse ¿̨ómo actúan ante este problema? En esta nota de investigación presentamos los resultados de un análisis crítico de los protocolos de prevención y de actuación frente a las violencias sexuales desarrollados por las universidades públicas catalanas, y ponemos particular atención en sus potencialidades y deficiencias. En el primer apartado, se introduce la investigación que sirve de marco para este estudio, el proyecto europeo «Apoyo a las víctimas de las violencias sexuales en el contexto universitario: creando modelos estables de formación (UsvReact)», así como la metodología empleada. Seguidamente, focalizamos la atención en la legislación que sirve de base para las políticas universitarias sobre violencias sexuales y de género para, finalmente, presentar los resultados del análisis de los protocolos. Este trabajo evidencia que las violencias sexuales en las universidades catalanas constituyen todavía un problema bastante desconocido y subestimado, al cual no se le ha dado la respuesta necesaria.

Palabras clave: violencias sexuales, violencias de género, universidad, política

\begin{abstract}
University campus is not a violence free space; therefore, how do universities deal with this problem? This research note presents the results of a critical analysis of prevention and action protocols against sexual violence in Catalan public universities. Particular attention is given to
\end{abstract}


the potentialities and shortcomings of such measures. The first section introduces the research that serves as a framework of this study. namely the European project "Universities Supporting Victims of Sexual Violence: Training for Sustainable Services (USVReact)", as well as the methodology used. Next, we focus our attention on the legislation in which university policies on sexual and gender violence are grounded. Finally, we present the results of our analysis of the protocols. This work shows that sexual violence in Catalan universities remains a quite unknown and underestimated issue that has not yet received the required response.

Keywords: sexual violence, gender-related violence, university, policy

\section{INTRODUCCIÓN}

En enero de 2017 aparece en la prensa una noticia que informa de la condena de siete años de cárcel a un catedrático de Sevilla por haber «molestado» a tres profesoras durante casi diez años (Europa Press, 2017). La Universidad, a pesar de conocer los hechos y ser declarada como responsable civil subsidiaria durante el juicio, no suspende al profesor, que sigue dando clase hasta después de la sentencia (Cela, 2017). Este es solo uno de los centenares de episodios de violencias que tienen lugar en las instituciones educativas de todo el mundo, y que solo en los últimos años han comenzado a salir a la luz gracias a las denuncias públicas realizadas por exestudiantes, especialmente en los países anglosajones. Sin embargo, aunque en el contexto español la mayoría de casos pasan todavía desapercibidos, hay ejemplos como este que empiezan a conocerse a través la prensa, lo que ha dado pie a amplios debates públicos. No obstante, las universidades siguen siendo percibidas como espacios no discriminatorios (Ballarín, 2015).

Quizás sea relevante recordar que en sus orígenes las universidades eran instituciones solo para varones, siguiendo así la herencia de los colegios catedralicios del siglo XII (Chuaqui, 2002), que vetaron el acceso a las mujeres hasta el siglo xIx (Palermo, 2006). Aunque con el paso del tiempo los códigos de género han evolucionado, se mantienen las estructuras de privilegios y las discriminaciones que de ellas se derivan (Ballarín, 2015; Biglia y Vergé, 2016). Es más, diferentes investigaciones internacionales evidencian la existencia de violencias de género en su interior (Gross et al., 2006; Feltes et al., 2012; Marshall, 2014; Phipps y Smith, 2012). Los pocos estudios realizados en España demuestran que la situación no es muy diferente (Bosch y Ferrer, 2000; Bodelón et al. 2012; Bardina y Murillo 2013). No obstante, el problema no solo no se reconoce, sino que, además, se invisibiliza, y cuando aparece en la prensa su interpretación se aleja de las relaciones de poder y dominación cotidianas presentes en el ámbito académico (Bosch et al., 2006).

Sin embargo, en las últimas décadas, en cumplimiento del marco legal (art. 23 a 25 de la Ley Orgánica 3/2007 para la Igualdad Efectiva entre Mujeres y Hombres; Ley Orgánica 4/2007, de 12 de abril, por la que se modifica la Ley Orgánica 6/2001, de 21 de diciembre, de Universidades; Ley Orgánica 2/2006, de 3 de mayo, de Educación) las universidades han creado unidades de igualdad con el objetivo de diseñar y 
poner en marcha políticas con enfoques de género. Actualmente, gracias a la presión de los movimientos feministas y al mayor debate público sobre violencias de género y sexuales, que se encuentran en la base del diseño de legislaciones europeas y nacionales que describimos en el tercer apartado, un número creciente de universidades espańolas ha adoptado medidas para prevenir y actuar ante discriminaciones y violencias de acuerdo con la previsión normativa. Entre ellas, las iniciativas más conocidas y comunes han sido los protocolos universitarios en materia de violencias sexuales, que serán objeto de nuestro análisis crítico. Nuestra finalidad en esta nota de investigación es comprender el contenido de estos protocolos, así como su implementación, a fin de valorar su efectividad; para ello, tenemos en cuenta la opinión las personas encargadas de su desarrollo. En último término, tratamos de reflexionar sobre la necesidad de mejorar las políticas universitarias sobre esta materia.

Desde el feminismo se insiste en que una respuesta efectiva al problema de las violencias sexuales y de género pasa necesariamente por reconocer y comprender su condición estructural. Por lo tanto, hay que tener en cuenta cómo se relacionan estas violencias con la cultura de la violación, los estereotipos de género y las relaciones de poder heterosexistas que existen en la base de la organización académica.

Partimos de una lectura de las violencias sexuales (VS) entendidas como el producto «sano» de la cultura heteropatriarcal que «reflejan y refuerzan las relaciones de poder jerárquicas e injustas» (Toledo y Pineda, 2016: 8). De esta manera, las VS constituyen una de las formas de control hacia las mujeres y hacia los sujetos sexualmente y/o genéricamente no normativos, y tienen su origen tanto en una visión estereotipada de los géneros como en las relaciones de poder (Biglia, 2015). Si bien en la legislación española se considera que cuando no hay una confrontación física fuerte no pueda hablarse de violencia, sino de abuso, creemos que esto constituye un enfoque reduccionista, que deja desamparadas y vuelve a violentar a la mayoría de las supervivientes de situaciones de violencia. En este sentido, consideramos necesaria una redefinición del término violencia que reconozca «que hay violencia sexual siempre que se atenta contra nuestra libertad sexual o que, a través de nuestra sexualidad se atenta contra nuestra libertad como sujetos» (Biglia y Jiménez, 2018: 153). Este es el enfoque a partir del cual desarrollamos nuestro análisis crítico. Tras esta breve introducción, destacamos aquellos aspectos metodológicos del proyecto europeo USVReact que están a la base del análisis usado en este texto. Seguidamente, a fin de entender el contexto legal en el que se producen las respuestas universitarias a las violencias sexuales, presentamos una revisión crítica de las normativas y de las políticas públicas en esta materia. En el apartado siguiente detallamos los resultados del análisis del abordaje de las violencias sexuales en las universidades catalanas desarrollado en el USVReact. Finalizamos esta nota con unas reflexiones acerca de la situación actual y algunas recomendaciones de cara al futuro. 


\section{METODOLOGÍA}

Las reflexiones que presentamos aquí forman parte del proyecto de investigación-acción europeo desarrollado entre 2016 y 2018, «Apoyo a las víctimas de las violencias sexuales en el contexto universitario: creando modelos estables de formación (UsvReact)», que tenía como objetivos diseñar, pilotar y evaluar modelos formativos dirigidos a facilitar que el personal universitario pudiera reconocer y dar una primera respuesta frente a situaciones de violencia sexual. Para ello, el análisis de las políticas universitarias en respuesta a las violencias sexuales, objeto específico de este texto, constituía un paso previo y necesario para el diseño de la formación a los y las docentes.

Nuestra investigación se enmarca en la epistemología del conocimiento situado y en las metodologías de investigación feministas (Biglia y Bonet 2017). El diseño de investigación es eminentemente cualitativo y exploratorio, por lo que no incluye una hipótesis que demostrar; sino que tiene por finalidad generar nuevo conocimiento a partir del análisis de una realidad todavía poco investigada. De hecho, de acuerdo con los principios de la investigación-acción, las especificidades del proceso de análisis se han ido refinando en interacción con las sensibilidades surgidas en el mismo proyecto. Para ello se han tenido en cuenta no solo las reuniones del equipo local, sino especialmente los debates sobre las políticas de las diferentes universidades socias y colaboradoras del proyecto ${ }^{1}$, así como las reuniones del «Grupo de trabajo con perspectiva feminista sobre violencias sexuales en las universidades» (GtFVsU) ${ }^{2}$. Finalmente, en este proceso de construcción-validación colectiva de conocimiento, también se han tenido en cuenta los debates surgidos a partir de las presentaciones de los resultados parciales realizadas en distintos espacios académicos (cuatro nacionales y cinco internacionales). Sustantivamente, nuestra investigación se encuadra en el campo de análisis y evaluación de políticas públicas con perspectiva de género. Siguiendo los principios explicitados por Bustelo (2014), hemos desarrollado una evaluación sumativa, ex post facto, centrada en el diseño y en los efectos de las políticas públicas. Para ello, en el apartado teórico nos hemos centrado en el análisis del contexto en que se generaron los protocolos evaluados, teniendo en cuenta el marco normativo nacional y autonómico; por otra parte, en el apartado empírico hemos situado nuestro foco en cómo las personas son afectadas por las políticas.

1. Brunel University (coord.), Universitat Rovra i Virgili, Universidad del País Vasco, University of Sussex, Universitá degli Studi di Torino, Panteion University of Social and Political Sciences (PUA), University of York y de las universidades colaboradoras que se pueden consultar en el siguiente enlace: http://usvreact.eu/es/associates/.

2. Se han realizado tres reuniones presenciales del grupo: el 17 de octubre de 2016, el 7 de julio de 2017 y el 26 de febrero de 2018, en las que han participado entre diez y veinte expertas en temas de género de diferentes universidades catalanas, muchas de las cuales habían tenido cargos relacionados con la gestión de las políticas de género en la universidad y/o habían realizado investigaciones al respecto. 
Para el desarrollo de esta fase de la investigación, que resumimos en esta nota de investigación, nos hemos basado en la siguiente pregunta generadora: ¿̨de qué manera las universidades están asumiendo su responsabilidad ante las violencias sexuales que se reproducen en las instituciones? Y los siguientes objetivos:

a) Conocer de qué manera ha influido el marco normativo en la elaboración e implementación de los protocolos y evidenciar sus límites partiendo de un enfoque de género.

b) Detectar similitudes y diferencias entre las políticas universitarias catalanas desarrolladas para dar respuesta a situaciones de acosos y violencias sexuales.

c) Identificar cuáles han sido las potencialidades y los límites en la formulación y diseño de los protocolos para hacer frente al problema de las violencias sexuales en las universidades.

d) Conocer cuáles han sido los puntos fuertes, las dificultades y los problemas observados en la implementación de los protocolos.

e) Ofrecer unas recomendaciones preliminares para su mejora desde la perspectiva de género.

Para alcanzar el primer objetivo hemos realizado una revisión bibliográfica que nos permite identificar y conocer los marcos legales estatales y autonómicos en los cuales se encuadran los protocolos. Así, si bien el Estado español carece de un marco normativo y de políticas públicas que garanticen la respuesta integral ante las violencias sexuales (Plataforma CEDAW Sombra Espańa, 2017), existen legislaciones relevantes a la hora de entender los protocolos universitarios y sus principales limitaciones. En el siguiente apartado de este artículo los presentamos de la mano de teóricas feministas.

La parte empírica de nuestro trabajo se ha basado principalmente en el análisis de contenido de los documentos legales, entrevistas a personas claves y reuniones con expertas para evaluar el análisis y las interpretaciones realizadas hasta el momento. La primera técnica nos ha servido, en particular, para alcanzar los objetivos b) y c), y parcialmente el e). Como corpus de análisis, hemos seleccionado todos los protocolos en vigor (a fecha diciembre 2017) en las ocho universidades de la Asociación Catalana de Universidades Públicas (ACUP) (tabla 1). La decisión de analizar estas normas no ha sido casual, en tanto que se trata de las únicas políticas desarrolladas para dar respuestas a los casos de violencias en las instituciones de enseñanza superior.

TABLA I.

Protocolos DE LAS UNIVERSIDADES PÚblicas CATALANAS*

\begin{tabular}{lccl}
\hline Universidad & Acrónimo & Año & \multicolumn{1}{c}{ Título } \\
\hline $\begin{array}{l}\text { Universitat } \\
\text { Autònoma de } \\
\text { Barcelona }\end{array}$ & UAB & 2016 & $\begin{array}{l}\text { Protocolo de actuación contra el acoso sexual y el } \\
\text { acoso por motivos de sexo, de orientación sexual, } \\
\text { identidad de género o expresión de género de la } \\
\text { Universitat Autònoma de Barcelona }\end{array}$ \\
\hline
\end{tabular}


.../...

\begin{tabular}{|c|c|c|c|}
\hline Universidad & Acrónimo & Año & Título \\
\hline $\begin{array}{l}\text { Universitat de } \\
\text { Barcelona }\end{array}$ & UB & 2014 & $\begin{array}{l}\text { Protocolo de la Universitat de Barcelona para la } \\
\text { prevención, la detección y la actuación contra las } \\
\text { situaciones de acoso sexual y por razón de sexo o } \\
\text { de orientación sexual }\end{array}$ \\
\hline $\begin{array}{l}\text { Universitat de } \\
\text { Girona }\end{array}$ & UdG & 2017 & $\begin{array}{l}\text { Protocolo de prevención y actuación ante } \\
\text { situaciones de violencia o acoso por razón de } \\
\text { sexo, género o sexualidad en la Universitat de } \\
\text { Girona }\end{array}$ \\
\hline $\begin{array}{l}\text { Universitat de } \\
\text { Lleida }\end{array}$ & UdL & 2013 & $\begin{array}{l}\text { Reglamento de actuación en caso de violencia de } \\
\text { género de la Universitat de Lleida }\end{array}$ \\
\hline $\begin{array}{l}\text { Universitat de } \\
\text { Vic-Central de } \\
\text { Catalunya }\end{array}$ & $\begin{array}{l}\text { UVIC- } \\
\text { UCC }\end{array}$ & 2015 & $\begin{array}{l}\text { Protocolo de prevención y abordaje del acoso } \\
\text { sexual y el acoso por razón de sexo o de } \\
\text { orientación sexual }\end{array}$ \\
\hline $\begin{array}{l}\text { Universitat } \\
\text { Oberta de } \\
\text { Catalunya }^{3}\end{array}$ & UOC & 2013 & $\begin{array}{l}\text { Protocolo contra el acoso sexual y por razón de } \\
\text { sexo u orientación sexual }\end{array}$ \\
\hline \multirow{2}{*}{$\begin{array}{l}\text { Universitat } \\
\text { Politècnica de } \\
\text { Catalunya }^{3}\end{array}$} & UPC-L & 2016 & $\begin{array}{l}\text { Protocolo de actuación y prevención del acoso } \\
\text { sexual y/o por razón de sexo, orientación sexual, } \\
\text { identidad de género o expresión de género en la } \\
\text { UPC }\end{array}$ \\
\hline & UPC-E & 2017 & $\begin{array}{l}\text { Protocolo de prevención y actuación contra el } \\
\text { acoso sexual y/o por razón de sexo, orientación } \\
\text { sexual, identidad de género o expresión de } \\
\text { género que afecten al estudiantado }\end{array}$ \\
\hline \multirow{2}{*}{$\begin{array}{l}\text { Universitat } \\
\text { Pompeu Fabra }\end{array}$} & UPF-E & 2015 & $\begin{array}{l}\text { Protocolo para la prevención, la detección y la } \\
\text { actuación contra la violencia machista que afecte } \\
\text { a estudiantes de la UPF }\end{array}$ \\
\hline & UPF-L & 2016 & $\begin{array}{l}\text { Protocolo para prevenir y solucionar conflictos } \\
\text { en materia de conductas violentas, } \\
\text { discriminatorias o de acoso }\end{array}$ \\
\hline $\begin{array}{l}\text { Universitat } \\
\text { Rovira i Virgili }\end{array}$ & URV & 2015 & Protocolo de actuación en caso de posible acoso \\
\hline
\end{tabular}

*A partir de ahora, por simplicidad, haremos referencia a estos protocolos a través del acrónimo de la universidad a la que pertenecen).

Fuente: elaboración propia.

3. Aunque de hecho la UOC funcione como si fuera una universidad pública a distancia y forme parte del ACUP, se trata de una fundación dirigida por un patronato integrado por diferentes entidades públicas y privadas, así como por personas físicas y jurídicas. Como se puede leer en su web «La UOC es una universidad que presta un servicio público y se organiza mediante gestión privada" (https://www.uoc.edu/portal/es/universitat/faqs/sobre-universitat/index.html). 
Los documentos seleccionados han sido examinados y comparados para detectar diferencias y similitudes, así como los puntos fuertes y débiles de los documentos, a través de un análisis de contenido en relación con las dimensiones detalladas en la tabla 2.

Nuestra lectura crítica ha tenido en cuenta los elementos que según Facio (2004) hay que respetar para facilitar la incorporación de la perspectiva de género en el derecho. Así, entre otros elementos, hemos indagado sobre la reproducción o manifestaciones de sexismo en el uso del lenguaje; la (in)capacidad para reconocer las especificidades de las diferentes personas pertenecientes a la comunidad universitaria a través de las maneras de identificar, nombrar y describir los sujetos; las visiones previas, mitos y creencias culturales que están en la base de estas normas; los mecanismos desarrollados en la práctica para apoyar a las personas que se sienten abusadas y evitar su victimización secundaria, y la asunción o ausencia de una perspectiva de género e interseccional.

TABLA 2.

Dimensiones ANALIZADAS

- Tipología

- Legislación mencionada

- Justificación y finalidad

- Descripción de los sujetos

- Terminología y lenguajes utilizados

- Medidas preventivas y formativas

- Tipo y formas de activación del procedimiento
- Ámbito de aplicación (personal/ relacional/territorial)

- Estructura responsable del procedimiento y su duración

- Medidas de intervención (cautelares/ correctoras/complementarias/ sancionatorias)

- Seguimiento

- Otros elementos relevantes

Fuente: elaboración propia

Sin embargo, este análisis resultaba insuficiente para entender cómo se habían desplegado tales medidas, sus articulaciones, sus fragilidades durante la aplicación y otros elementos clave a la hora de determinar su funcionamiento - objetivos d) y e)—.

Por esta razón, entre abril de 2016 y noviembre del mismo año, realizamos doce entrevistas semiestructuradas a expertas de diferentes universidades catalanas. Como muestra intencional, escogimos a las personas responsables de la implementación de los protocolos en la UB, la UdL, la UVIC-UCC, la UPC, la UPF y la UAB. En el caso de la URV, al no existir un protocolo específico, decidimos investigar más en profundidad y seleccionamos una delegada sindical que estaba luchando para que se elaborara un protocolo específico, así como una persona de referencia del protocolo de acoso laboral que tenía perspectiva de género y una responsable para cuestiones de género de la Universidad. Además, en la UAB entrevistamos a la anterior responsable, ya que la persona que en ese momento ejercía el cargo nos remitió a ella, así como a una técnica que se dedicaba a la atención directa, la única con esa atribución en las universidades públicas catalanas. En 2018, tuvimos la oportunidad de entrevistar también a la responsable de la UdG, ya que no nos había sido posible concertar 
anteriormente una cita con su predecesora. De esta forma, alcanzamos un total de trece entrevistas. Desafortunadamente, no pudimos incluir en la muestra ninguna responsable de la UOC por falta de disponibilidad. Estas entrevistas nos sirvieron para conocer tanto los procesos de generación de los protocolos y su eventual relación con otras políticas universitarias, como para entender aquellos elementos que no habían quedado suficientemente explícitos en los mismos, su eventual implementación, así como las dificultades encontradas en el proceso. Las dimensiones de las entrevistas quedan resumidas en la tabla 3.

TABLA 3.

DimENSIONES ENTREVISTAS

- Políticas de prevención y actuación.

- Proceso de diseño y aprobación, implementación, rol de las unidades de igualdad en la implementación/control de las políticas, límites, puntos fuertes y posibles mejoras.

- Medidas preventivas (comunicación, difusión y sensibilización) implementadas o que se prevén desarrollar en la práctica.

- Medidas formativas.

Fuente: elaboración propia.

En el cuarto apartado de este artículo presentamos los resultados entrelazados de estas dos últimas fases de análisis, incorporando elementos surgidos en los debates mantenidos con otras expertas tanto en las reuniones del GtFVsU, como en los otros fórums académicos en los que hemos presentado los resultados parciales.

\section{LOS MARCOS QUE MARCAN}

Tal y como anunciamos anteriormente, en este apartado expondremos someramente los ordenamientos jurídicos que enmarcan específicamente el desarrollo de las normas universitarias analizadas.

TABLA 4 .

LEGISLACIÓN DE REFERENCIA

a. Artículos 178-194 de la Ley Orgánica 10/1995, de 23 de noviembre, del Código Penal

b. Ley Orgánica $1 / 2004$, de 28 de diciembre, de medidas de protección integral contra la violencia de género

c. Ley 5/2008, de 24 de abril, del derecho de las mujeres a erradicar la violencia machista del Parlament de Catalunya

d. Ley Orgánica 3/2007, de 22 de marzo, para la igualdad efectiva de mujeres y hombres

e. Ley $11 / 2014$, de 10 de octubre, para garantizar los derechos de lesbianas, gays, bisexuales, transgéneros e intersexuales y para erradicar la homofobia, la bifobia y la transfobia del Parlament de Catalunya

Fuente: elaboración propia 
La «Ley Orgánica 3/2007, de 22 de marzo, para la igualdad efectiva de mujeres y hombres», y la "Ley 11/2014, de 10 de octubre del Parlament de Catalunya, para garantizar los derechos de lesbianas, gais, bisexuales, transgéneros e intersexuales y para erradicar la homofobia, la bifobia y la transfobia», han tenido una influencia directa en la elaboración de los protocolos examinados. Las otras tres legislaciones a las que hacemos referencia han servido como fundamento para su desarrollo ulterior, como queda evidenciado en los preámbulos de los documentos analizados.

Siguiendo el análisis realizado por politólogas y juristas, describimos brevemente para cada uno de estos textos legislativos algunos de los elementos críticos/criticables desde una perspectiva feminista y sus principales relaciones con las normas universitarias.

\section{El Código Penal (CP)}

Los protocolos universitarios se basan en los arts. 178-194 del CP, en los cuales se definen las diferentes formas de violencias (acoso sexual, agresión sexual...). Ahora bien, tal y como nos recuerdan Toledo et al., (2016), durante el siglo xx y, en particular, a partir de la denominada "segunda ola feminista», se ha ido cuestionado fuertemente el modelo jurídico penal basado en «los delitos contra la honestidad» y la «moral sexual», lo que ha llevado a sucesivas reformas de los códigos penales para incorporar los delitos contra la libertad sexual; por ejemplo, a través de la aprobación de la Ley Orgánica 10/1995. De este modo, las agresiones sexuales, los abusos sexuales, los delitos de exhibicionismo, de provocación sexual y los delitos relativos a la prostitución han quedado incluidos en el capítulo denominado «Delitos contra la libertad sexual». Con ello, se ampliaba el catálogo de conductas punibles relacionadas con las violencias sexuales; se endurecían las penas, principalmente, para mejorar la protección penal de las personas menores de edad, y se reconocía que el sujeto ofendido podía ser un hombre.

No obstante, dicha reforma ha sido criticada por la centralidad que mantienen todavía la penetración y el consentimiento en la distinción de los tipos delictivos (Toledo et al., 2016). De hecho, la violación continúa siendo considerada un crimen moralizador (Segato, 2003) y los delitos sexuales se perciben de menor gravedad si no implican una penetración forzada, reforzando así la creencia de que la violencia sexual es equivalente a la violación con penetración, lo que rebaja los otros tipos de violencias a meras molestias (Fernández Díaz, 2003). Por otro lado, un mal uso de la noción de consentimiento marcaría la diferencia entre una agresión, con uso de la violencia física o intimidación, y un abuso sexual. Sin embargo, tal y como afirma Montse Pineda en una entrevista, "poner el foco en el consentimiento es demostrar que no lo has dado. Esto hace que muchos abogados, y a veces los magistrados, pregunten de qué manera lo hiciste, cómo te resististe, si opusiste fuerza, si gritaste o pediste ayuda» (Borraz, 2017). 


\section{La violencia de género}

Muchos protocolos analizados se basan en la Ley Orgánica 1/2004, que tiene como objetivo actuar contra la violencia de género entendida como «una manifestación de discriminación, de una situación de desigualdad y relaciones de poder del hombre sobre la mujer» (art. 1.1). Sin embargo, en sus cláusulas más prácticas limita esta comprensión de la violencia de género a aquella que es ejercida por un hombre «hacia quienes son o han sido sus cónyuges o quienes están o han sido vinculadas a ellos por relaciones similares de afecto, con o sin convivencia, incluyendo cualquier acto de violencia física y psicológica» (art. 1.3). Así, se invisibilizan otras formas de violencia y se brindan medidas de protecciones integral solo en el caso de violencias entre parejas y exparejas heterosexuales (Bonet, 2007; Larrauri, 2007; Bustelo y Lombardo, 2012). En palabras de la filósofa Celia Amorós (2008), estamos ante una "chapuza conceptual» que despolitiza, confunde e impide llevar a cabo un abordaje jurídico-político adecuado. Se ha realizado una metonimia sustituyendo la violencia de género (el todo) por la violencia contra las mujeres en las relaciones de pareja heterosexuales (la parte) (Bustelo y Lombardo, 2012). Este reduccionismo produce desigualdad en el nivel de protección y acceso a los servicios por parte de las supervivientes. Así, por ejemplo, el juicio sobre la violación múltiple que tuvo lugar durante los Sanfermines de 2016, más conocido como el caso de La Manada, al no ser considerado como violencia de género se celebró en Pamplona, obligando a la superviviente a desplazarse para declarar, mientras que en los casos amparados por la Ley 1/2004 se prioriza el domicilio de la víctima como lugar para el juicio.

Por lo que concierne al argumento central de esta nota de investigación, es preciso recordar que en la Ley 1/2004 se reconoce que las universidades tienen que asumir determinadas responsabilidades que «incluirán y fomentarán en todos los ámbitos académicos la formación, docencia e investigación en igualdad de género y no discriminación» (art. 7). Sin embargo, la ley no define cómo debe ser el proceso de rendición de cuentas — en relación con estas responsabilidades — por parte de las instituciones de enseñanza superior, ni estipula los recursos humanos y económicos necesarios para su puesta en práctica. En este sentido, a pesar de que las acciones de prevención, sensibilización e intervención en violencia de género están generalmente incluidas en los planes de igualdad, muchas veces no reciben el suficiente apoyo institucional para ser implementadas, lo que acaba resultando en diferencias notorias entre las diferentes universidades, tanto en relación con el ámbito de la docencia como en el de la investigación (Verge y Cabruja, 2017).

\section{Igualdad}

La «Ley Orgánica 3/2007 de 22 de marzo para la igualdad efectiva de mujeres y hombres», que transpone la «Directiva 2002/73/CE del Parlamento Europeo relativa a los principios de igualdad de trato entre hombres y mujeres en lo que se refiere al 
acceso al empleo, la formación y la promoción profesional», obliga a «la prevención del acoso sexual y del acoso por razón de sexo [...] las Administraciones públicas negociarán $[\ldots]$ un protocolo de actuación que comprenderá [...] el compromiso [...] de los organismos públicos [...] de prevenir y no tolerar el acoso sexual y el acoso por razón de sexo» (art. 62). La misma ley, en su título IV («El principio de igualdad en el empleo público»), se ocupa del derecho al trabajo en igualdad de oportunidades e incluye, entre los derechos laborales, la protección frente al acoso sexual y por razón de sexo. El límite principal de esta norma deriva de la incorrecta o parcial aplicación del principio en el que dicen basarse: el denominado principio de mainstreaming de género. En el contexto español, se ha traducido como mera transversalización, muchas veces focalizándolo simplemente en la igualdad de oportunidades en la esfera laboral (Salazar, 2018). De acuerdo con Juana Gil (2012: 17) «para conseguir los objetivos perseguidos por el gender mainstreaming requiere cambios catárticos, no conseguibles de manera inmediata, a saber: revisión profunda de la gestión de las políticas, compromiso serio con la igualdad efectiva y formación en género de todo el personal implicado en su puesta en marcha».

\section{Violencias machistas}

La Ley catalana 5/2008 de 24 de abril utiliza una definición más amplia que la legislación estatal al definirla como violencia machista. Algunos protocolos, como el de la UPF-E, se fundamentan en esta ley para definir las dimensiones de las violencias que quieren abordar. De hecho, esta ley reconoce las diferentes manifestaciones de las violencias de género y establece medidas integrales para la prevención, la asistencia y la protección de la recuperación y reparación para todas las supervivientes. No obstante, igual que sucede con la norma estatal, acaba solo reconociendo la violencia contra las mujeres e invisibiliza otras violencias de género causadas por la sociedad heteropatriarcal (Biglia, 2015).

\section{LGBTIfobia}

Más reciente es la «Ley 11/2014 de 10 de octubre del Parlament de Catalunya, para garantizar los derechos de lesbianas, gais, bisexuales, transgéneros e intersexuales y para erradicar la homofobia, la bifobia y la transfobia». Su objetivo es garantizar el derecho a la igualdad y la no discriminación de cualquier persona independientemente de su orientación sexual, opción afectivo-sexual o identidad de género. En ella se reconocen diversas formas de violencia, como la discriminación y las violencias por razón de la orientación sexual, por identidad de género o por expresión de género (art. 9.1), la homofobia (art. 26.3), la transfobia (art. 26.3) y la bifobia (art. 26.3). También se incluyen conceptos como la violencia hacia las personas LGBTI (art. 6), las agresiones y las discriminaciones contra las personas LGBTI (art. 39.2), el acoso (art. 12.6) y la exclusión (art. 12.6). Además, se establecen los medios y las medidas que se deben aplicar para la consecución de estos derechos en los ámbitos en los que las autoridades 
públicas de Cataluña tienen poder, ya sean estos públicos o privados. Así, en su art. 13 se obliga a las universidades catalanas a promover medidas de protección, de apoyo y de investigación para aumentar la visibilidad de las personas LGBTI, así como el desarrollo de medidas para la no discriminación y elaboración de un protocolo específico.

Estas leyes han marcado el desarrollo de los protocolos de prevención y actuación frente a las violencias sexuales en las universidades catalanas. Pero, ¿cómo se han puesto en práctica las obligaciones y las recomendaciones legales? ¿De qué manera estas políticas dan respuesta al complejo problema de las violencias y abusos sexuales en el entorno universitario? A estas y otras preguntas intentaremos contestar en el próximo apartado.

\section{MIRANDO CON LUPA LOS PROTOCOLOS DE LAS UNIVERSIDADES PÚBLICAS CATALANAS}

\section{Habemus protocolo}

Como se puede ver en la tabla 1, la mayoría de las universidades catalanas (UB, UAB, UVIC-UCC, UPC, UdG) disponen de un protocolo para el acoso laboral, y otro más específico sobre las violencias de género, machistas, sexuales y/o LGTBIfóbicas, lo que otorga reconocimiento específico a estas últimas. Sin embargo, se evidencia que hay universidades (UPF-L/UPF-E) que no están todavía adaptadas a los requisitos de la Ley 11/2014, o que solo lo están parcialmente, como la UdL o UOC, tal y como se expondrá seguidamente ${ }^{4}$. Por una parte, la URV y la UPF-L no disponen de protocolos específicos, por lo cual hemos examinado las medidas previstas en casos de acoso en la primera universidad, y las medidas previstas para conductas violentas, discriminatorias o de acoso en el caso de la segunda universidad, a fin de comprobar cómo se aplicarían en las circunstancias que nos incumben. Por otra parte, la UPF y la UPC poseen dos protocolos, uno orientado a la protección del estudiantado (UPF-E, UPCE) y el otro a la protección del personal docente y administrativo (UPF-L, UPC-L). Finalmente, el resto de universidades (UAB, UVIC-UCC, UPF, UPC) han aprobado y modificado estos protocolos al menos dos veces para adaptarlos a las obligaciones legales o para aportar algunas mejoras 5 .

Tal y como se evidenció en las entrevistas realizadas, solamente en tres casos el diseño de los protocolos universitarios catalanes fue encargado a equipos o personas

4. Abordamos el análisis del problema de la LGTBI fobia en las universidades catalanas en Biglia y Cagliero (en prensa).

5. Por ejemplo, mientras acabábamos este artículo se ha aprobado un nuevo protocolo integral en la UPF; en la UB han empezado el rediseño de su instrumento, para lo que hemos sido interpeladas como consultoras externas, y la URV se encuentra en pleno proceso participativo para crear un protocolo que incluya estudiantes e mejorar el de personal laboral 
especializadas en género o que dispusieran de competencias específicas en el tema. En cambio, en relación con los procesos de escritura y revisión, se observa una mayor influencia de los gabinetes jurídicos de las universidades, que han imprimido su huella. Tampoco se evidencia que los protocolos hayan sido diseñados a través de procesos participativos orientados a detectar y responder a las necesidades de toda la comunidad universitaria, excepto en el caso de la UdL y de la UdG. Es más, su existencia es desconocida por la gran mayoría de la comunidad universitaria, como ya han evidenciado algunos estudios (Bodelón et., 2012) y como confirman nuestras informantes.

Por tanto, resulta complicado evaluar la efectividad de los protocolos, ya que la mayoría de universidades espańolas, contrariamente a lo que ocurre por ejemplo en Estados Unidos, no sistematizan las demandas recibidas ni realizan una evolución del proceso. Así, la periodista de investigación Yuly Jara, que ha realizado la única infografía actualmente disponible al respecto ${ }^{6}$, explicaba en el «I Congreso Mujeres y Universidad sin Cifras. La Violencia Invisible» ${ }^{7}$ las reticencias de muchas universidades a compartir con ella los datos anonimizados sobre la aplicación de los protocolos. En ese mismo encuentro, las responsables de los observatorios de género de varias universidades expresaron su preocupación por difundir informaciones sobre los casos, por el peligro de generar victimización secundaria. En este sentido, previo acuerdo de confidencialidad, hemos debatido con nuestras informantes sobre las situaciones de violencia vividas en sus universidades, a fin de detectar las posibilidades y límites de los protocolos. Para salvaguardar dicha confidencialidad, solo podemos comentar que, en el momento de realización de las entrevistas, en diferentes universidades catalanas tales protocolos se habían activado muy pocas veces, y en el caso de la UAB, la UdL, la UB y la UdG, UPC nunca.

Este dato no resulta sorprendente, teniendo en cuenta que si bien la mayoría de protocolos hace referencia a quién hay que contactar para recibir asesoría sobre el procedimiento, en ninguno de ellos se especifica la presencia de servicios para las personas que se sienten acosadas. En palabra de una de nuestras entrevistadas "no siempre queda demasiado claro quién puede atender casos de violencias". El único asesoramiento que explícitamente se ofrece es para la apertura de un expediente, y con frecuencia las personas necesitan dar sentido a sus propias vivencias antes de decidir si empezar un proceso de este tipo. Además, si bien la mayoría de documentos — excepto en los casos de la UdL, la UdG y la UOCestablecen un tiempo máximo para resolver los procedimientos, las entrevistadas apuntan a que en las pocas ocasiones que se han activado, las actuaciones institucionales han sido lentas, opacas y escasamente empáticas con las persones denunciantes.

¿Cómo es posible que políticas diseñadas ad hoc se hayan activado solo muy de vez en cuando? En opinión de las entrevistadas, una de las razones residiría en que la red entre las personas/oficinas involucradas es menos efectiva y eficaz de lo que se necesitaría. Sin embargo, tal y como mostraremos más adelante, estos límites se refieren

6. Disponible en: http://bit.ly/2xCmq1s.

7. Valencia, 21-23 de noviembre de 2018. Disponible en: http://bit.ly/32gng1V. 
también a la naturaleza de las políticas y la forma de abordar las violencias sexuales que plantean. En este sentido, es preciso recordar que no estamos ante políticas públicas de amplio alcance, sino ante documentos rígidos y procedimentales que no solo reproducen sino también impactan en la lectura e interpretación de las violencias sexuales de la comunidad universitaria.

\section{TABLA 5 .}

FiNALIDADES Y TIPIFICACIÓN DE LAS VIOLENCIAS EN LOS PROTOCOLOS DE LAS UNIVERSIDADES PÚBLICAS CATALANAS Y LA UOC EN VIGOR EN FEBRERO 2018

Dimensiones

Enf. Finalidades
Def. probl.

violencias

\begin{tabular}{|c|c|c|c|c|}
\hline \multirow{3}{*}{ 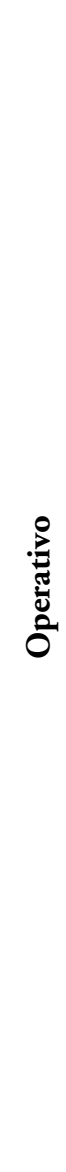 } & UB & $\begin{array}{l}\text { «Informar, formar y sensibilizar } \\
\text { [...]. Disponer de la organización } \\
\text { específica y determinar } \\
\text { procedimientos ágiles de } \\
\text { intervención y de acompañamiento } \\
\text { [...]. Garantizar la seguridad, la } \\
\text { integridad y la dignidad de las } \\
\text { personas afectadas [...]. Garantizar } \\
\text { la confidencialidad de las personas } \\
\text { afectadas» (p. 4). }\end{array}$ & Estructural & $\begin{array}{l}\text { Acoso (sexual, por } \\
\text { razón de género, por } \\
\text { orientación sexual) }\end{array}$ \\
\hline & UPF-L & $\begin{array}{l}\text { «Establecer los criterios esenciales, } \\
\text { de carácter mínimo, para gestionar } \\
\text { las conductas percibidas como } \\
\text { discriminatorias, violentas o de } \\
\text { acoso dentro del ámbito de } \\
\text { dirección y de organización de la } \\
\text { unidad administrativa o académica } \\
{[\ldots . .] \text { (p.2). }}\end{array}$ & Laboral & $\begin{array}{l}\text { Conducta: de acoso } \\
\text { laboral, violenta en el } \\
\text { lugar de trabajo, } \\
\text { discriminatoria } \\
\text { (razón de sexo, } \\
\text { orientación u opción } \\
\text { sexual, por embarazo } \\
\text { y maternidad), de } \\
\text { acoso sexual en el } \\
\text { trabajo }\end{array}$ \\
\hline & URV & $\begin{array}{l}\text { "(El protocolo) quiere asegurar un } \\
\text { procedimiento interno, a través del } \\
\text { cual la URV pueda protocolizar la } \\
\text { comunicación, el estudio, la } \\
\text { resolución y la sanción, si es } \\
\text { necesario, ante de un posible caso } \\
\text { de acoso que se produzca en la } \\
\text { comunidad universitaria» (p.3). }\end{array}$ & Laboral & $\begin{array}{l}\text { Acoso (laboral, } \\
\text { sexual, por razón de } \\
\text { género, orientación } \\
\text { sexual, psicológica, } \\
\text { racista, religiosa...). }\end{array}$ \\
\hline
\end{tabular}


$\ldots / \ldots$

Enf. Finalidades Def. probl. $\begin{gathered}\text { Dimensiones } \\ \text { consideradas de las } \\ \text { violencias }\end{gathered}$

\begin{tabular}{|c|c|c|c|c|}
\hline & UAB & $\begin{array}{l}\text { «Responder a la medida } 4.5 \text { del } \\
\text { tercer plan de acción para la } \\
\text { igualdad entre mujeres y hombres } \\
\text { de la UAB (2013-2017): "Diseńar e } \\
\text { implementar una propuesta integral } \\
\text { de erradicación de las violencias de } \\
\text { género y las relaciones abusivas, } \\
\text { incrementando la coordinación } \\
\text { institucional, que incluya un } \\
\text { protocolo y un circuito de denuncia } \\
\text { y de atención en los casos de acoso } \\
\text { sexual y de acoso por razón de sexo } \\
\text { o de orientación sexual”» (p. } 3 \text { ). }\end{array}$ & Estructural & $\begin{array}{l}\text { Acoso sexual, por } \\
\text { razón de género, por } \\
\text { orientación sexual }\end{array}$ \\
\hline 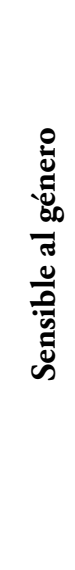 & UdL & $\begin{array}{l}\text { «Hacer efectiva esta igualdad (entre } \\
\text { mujeres y hombres), eliminando las } \\
\text { discriminaciones y las situaciones de } \\
\text { acoso sexual, acoso por razón de sexo } \\
\text { y de orientación sexual, y/o violencia } \\
\text { en el ámbito de la pareja que se } \\
\text { puedan producir a la UL» (p. 147). }\end{array}$ & Estructural & $\begin{array}{l}\text { Violencia de género } \\
\text { en el ámbito laboral } \\
\text { y académico. Acoso } \\
\text { sexual, por razón de } \\
\text { sexo o de orientación } \\
\text { sexual. } \\
\text { Discriminación } \\
\text { directa/indirecta por } \\
\text { razón de sexo y de } \\
\text { orientación sexual. } \\
\text { No indemnidad ante } \\
\text { de las represalias, } \\
\text { violencia en el } \\
\text { ámbito de la pareja }\end{array}$ \\
\hline
\end{tabular}

«La voluntad de la UOC es

reafirmar [con este protocolo] el

respeto por los derechos

UOC fundamentales de las personas y su preocupación de promover una comunidad universitaria y un

Acoso sexual, por

Estructural razón de género u

orientación sexual

ambiente de convivencia libre de

cualquier violencia» (p. 2). 
.../

Enf.

Finalidades

Def. probl.

Dimensiones

consideradas de las

violencias

\begin{tabular}{|c|c|c|c|c|}
\hline \multirow{5}{*}{ 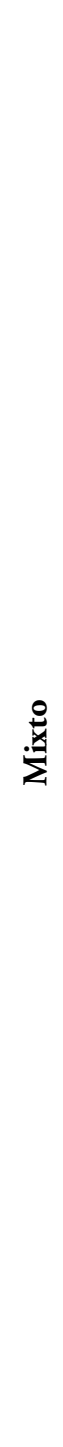 } & UdG & $\begin{array}{l}\text { «Prevenir la violencia y el acoso } \\
\text { entre los miembros de la } \\
\text { comunidad universitaria y } \\
\text { establecer un procedimiento de } \\
\text { actuación para detectarlos» (p. 2). }\end{array}$ & Estructural & $\begin{array}{l}\text { Violencia machista, } \\
\text { acoso sexual, por } \\
\text { razón de sexo, género } \\
\text { o sexualidad }\end{array}$ \\
\hline & UPC-E & $\begin{array}{l}\text { «Definir el marco de actuación en } \\
\text { relación con los casos que afecten al } \\
\text { estudiantado de la UPC con la } \\
\text { finalidad explícita de erradicar este } \\
\text { tipo de conductas en el ámbito de } \\
\text { la UPC» (p. 6). }\end{array}$ & Estructural & $\begin{array}{l}\text { Acoso, acoso sexual, } \\
\text { por razón de sexo, } \\
\text { orientación sexual, } \\
\text { identidad de género } \\
\text { o expresión de } \\
\text { género }\end{array}$ \\
\hline & UPC-L & $\begin{array}{l}\text { «Erradicar este tipo de conductas } \\
{[\ldots . .] \text { y ser una guía por la }} \\
\text { prevención, la detección y } \\
\text { resolución de estos casos» (p. 2). }\end{array}$ & Estructural & $\begin{array}{l}\text { Acoso (sexual y/o } \\
\text { por razón de sexo, } \\
\text { orientación sexual, } \\
\text { identidad de género } \\
\text { o expresión de } \\
\text { género) }\end{array}$ \\
\hline & UPF-E & $\begin{array}{l}\text { «El objetivo del protocolo es doble: } \\
\text { a) Prevenir la aparición de la } \\
\text { violencia machista entre los } \\
\text { miembros de la comunidad } \\
\text { universitaria. b) Establecer un } \\
\text { procedimiento de actuación para } \\
\text { detectar conductas [...], la } \\
\text { investigación de estas, si procede, la } \\
\text { propuesta de incoación de } \\
\text { expediente disciplinario [...]» (p. 2). }\end{array}$ & Estructural & $\begin{array}{l}\text { Violencia machista } \\
\text { (física, psicológica, } \\
\text { sexual y abusos } \\
\text { sexuales, agresiones } \\
\text { sexuales y acoso } \\
\text { sexual), conductas } \\
\text { sexistas }\end{array}$ \\
\hline & $\begin{array}{l}\text { UVIC- } \\
\text { UCC }\end{array}$ & $\begin{array}{l}\text { «Quiere conseguir un entorno de } \\
\text { trabajo saludable, productivo, } \\
\text { seguro y respetuoso para toda la } \\
\text { comunidad universitaria» (p.4). }\end{array}$ & Laboral & $\begin{array}{l}\text { Acoso sexual, acoso } \\
\text { por razón de sexo o } \\
\text { de orientación sexual }\end{array}$ \\
\hline
\end{tabular}

Fuente: elaboración propia.

Si analizamos el objetivo general (véase la primera columna de la tabla 5) podemos confirmar tres enfoques diferentes: 
- El primer enfoque es totalmente operativo, es decir, se centra en el proceso de actuación y se evidencia en los protocolos de la URV, UPF-L, UB. Sin embargo, en los casos de la UB y la UPF-L se reconoce la diversidad estructural de las violencias sexuales y de género, mientras que en la URV se invisibilizan las diferencias entre las tipologías de acoso.

- El segundo enfoque, compartido por la UOC, la UdL y la UAB parece más sensible al género y pretende definir instrumentos específicos para erradicar las violencias sexuales. Entre ellos, solo el protocolo de la UAB reconoce explícitamente el acoso por expresión de género, mientras que el de la $\mathrm{UdL}$ es el que menciona explícitamente los diferentes tipos de violencias, además del acoso por identidad de género o expresión de género, que se pueden dar en el ámbito universitario, a diferencia del de la UOC, que se focalizaría únicamente en los casos de acoso.

- El último enfoque, compartido la UPF-E, la UPC-L, la UPC-E y la UdG, correspondería al tipo que hemos denominado mixto; es decir, en él están presentes características de los otros dos enfoques. Así, el protocolo de la UdG sería el que ofrece una definición más amplia del problema al incorporar la violencia machista y el «acoso por sexualidad»; en cambio, el de la UPC se focalizaría únicamente en los casos de acoso, mientas que el de la UPF-E sería el único que definiría de forma manifiesta las conductas sexistas como una expresión de las violencias sexuales. Entre estos protocolos, los de la UPF-E y de la UdG serían los únicos que incorporan una perspectiva de definición de la violencia machista.

- Un caso particular correspondería al protocolo de la UVIC-UCC, que no podemos situar en ninguno de los tres enfoques propuestos porque existe una disonancia entre el objetivo declarado, de seguridad y bienestar en el lugar de trabajo, en relación con las dimensiones de las violencias consideradas, que se centran en formas específicas de violencias sexuales y no en casos de acoso laboral como podría esperarse.

Esta disimilitud de enfoques se refuerza a través de la legislación (internacional, comunitaria, estatal o autonómica) en que se basan los diferentes documentos. El hecho de que la mayoría adopte una visión amplia es motivo de esperanza para el desarrollo de abordajes más integrales en torno a la violencia de género; sin embargo, de acuerdo con el análisis lingüístico efectuado, evidenciamos que existen otros elementos que limitan esta lectura optimista

En primer lugar, y en contraste con la legislación española y la catalana, todos los sujetos mencionados en estas normativas están descritos con un lenguaje neutral respecto al género, utilizando sustantivos colectivos no sexuados (por ejemplo, comunidad universitaria) o nombres abstractos a la hora de ser descritos (la persona agresora, la persona acosada etc.). Esta supuesta neutralidad, que también encontramos, por ejemplo, en la legislación del Reino Unido (Alldred y Biglia, 2015), puede ser interpretada como la voluntad de adoptar un enfoque no discriminador. Sin embargo, esta forma de describir los sujetos conlleva el posible sobreentendido que hombres y mujeres pueden sufrir violencia sexual por igual, sin tener en cuenta las asimetrías 
estructurales de las relaciones de género (Cagliero y Biglia, 2016) ni las relaciones de poder jerárquicas intrínsecas a los entornos académicos.

En nuestra opinión, este uso de un lenguaje supuestamente neutral es una muestra de la resistencia por parte de las instituciones a reconocer la jerarquización y el reparto de poderes presentes en su seno. Tal y como sugieren Igareda y Cruells (2014: 9), sería conveniente "poner énfasis en las definiciones y conceptos de género para legislar en función de la categoría género, [...] utilizar nuevos conceptos jurídicos para traducir la situación de opresión que sufren las mujeres en función de diversas desigualdades, y seguir haciendo ciertos reclamos jurídicos cuando fuera necesario en base a un sujeto específico como el de "mujeres"». En cambio, esta negación (por omisión) del género, tal y como hemos analizado en los materiales creados para nuestra formación (Luxán et al., 2018), impide ofrecer respuestas adecuadas frente a situaciones de desamparo en las que las violencias sexuales están marcadas por unas fuertes disimetrías de poder.

En la misma línea, la mayoría de textos utilizan reiteradamente la expresión «víctima» (URV, UB, UdL, UPF-L, UPF-E, UPC-E, UOC, UdG) para describir a la persona acosada. El uso de esta expresión ofrece una interpretación pasiva de los sujetos que sufren violencia, y por lo tanto justifica la suplantación de su opinión y de su agencia a la hora de tomar decisiones procedimentales. De hecho, la rigidez de los protocolos analizados ofrece poco espacio al diseño de un camino personalizado por parte de las supervivientes, término que preferimos, en tanto permite resaltar la capacidad de superación y evitar construir identidades de pasividad, sometimiento o poca capacidad de defensa (Velázquez, 2003).

Finalmente, aunque en muchos de los objetivos que se plantean en los protocolos (como por ejemplo en la UdG, UPC-L, UPF-E) se reconoce la importancia de la prevención, es justamente en las medidas educativas de carácter integral (Bustelo y Lombardo, 2007) donde más se evidencia su ausencia. A pesar de que todos los documentos prevén realizar tanto acciones de difusión de los protocolos como campañas de sensibilización sobre acoso y violencias, con frecuencia, se trata únicamente de referencias abstractas, como por ejemplo "la difusión del protocolo a toda la comunidad universitaria, que será accesible en cualquier momento" (art. 8, pag.10 UVIC-UCC), sin detallar cuáles serán las intervenciones que realizar, los recursos que se destinarían a ellas y los mecanismos de evaluación concernidos. Únicamente en los casos de la UdG y la UPF-L se explicitan determinadas acciones preventivas y formativas. La UdG, por ejemplo, determina tres ejes alrededor de los cuales se desarrollará la prevención (art. 4):

- Información, sensibilización y formación para PDI, PAS e estudiantes etc.

- Evaluación del entorno laboral e identificación de los casos de acoso.

- Asesoramiento e información, a cargo de la Unidad de Igualdad.

A estas circunstancias cabe añadir que con frecuencia las medidas no especifican quiénes tienen la responsabilidad de implementarlas (UPC-L/UPC-E, URV, UAB). En este sentido, es relevante el caso de la UOC, que explicita que todas las personas trabajadoras, sin formación previa ni recursos de tiempo dedicado a ello, son igualmente responsables de la prevención y de garantizar un entorno laboral en el que se respete la 
dignidad de toda la comunidad universitaria. En otros casos, como en la UdL, existen centros específicos, como el Dolors Piera, encargados de las acciones de prevención, sensibilización y las investigaciones sobre el tema. Finalmente, únicamente tres protocolos reconocen la importancia de efectuar estudios y publicaciones alrededor de las violencias sexuales y de género (UPF-T, UPC-T, UB). También en este caso es frecuente que este tema no esté muy desarrollado. Así, la UPF-T y la UPC-T solo mencionan la importancia de realizar investigaciones para conocer la incidencia de estas conductas, sin ofrecer detalles sobre cómo ni quién se encargará de llevarlos a cabo. Solo la UB explica que las oficinas encargadas son la Unidad de Igualdad, el Servicio de Atención al Estudiante (SAE) y la Oficina de Seguridad, Salud y Medio Ambiente (OSSMA), y que el objetivo general de "indagar sobre la posible percepción o conocimiento de conductas constitutivas de acoso sexual o de acoso por razón de sexo en el ámbito de la Universidad, y sobre las medidas formativas y preventivas que se hayan implantado" (X.3.d) se tendrán que realizar como mínimo cada tres años.

Por otra parte, es innegable que existen medidas preventivas previstas en los planes de igualdad universitarios. De acuerdo con la ley estos constituyen un "conjunto ordenado de medidas, adoptadas después de realizar un diagnóstico de situación, tendentes a alcanzar en la empresa (universidad) la igualdad de trato y de oportunidades entre mujeres y hombres y a eliminar la discriminación por razón de sexo» (art. 46 de la Ley Orgánica 3/2007), y fijan los objetivos concretos que deben alcanzarse, así como las estrategias y prácticas que hay que adoptar. Si bien, en algunos casos, se contemplan acciones contra el acoso y las violencias sexuales, tan solo algunas universidades catalanas (UAB, UPF, UB, URV, UdL) han desarrollado jornadas, formación no reglada o campańas de sensibilización. Además, antes de las formaciones específicas sobre violencias sexuales y de género desarrolladas en el marco de nuestro proyecto (USVReact), solo se habían realizado cursos reconocidos de forma puntual en la UAB y en la UdL orientados al personal y los estudiantes. Así, de acuerdo con lo expresado por nuestras informantes, la necesidad de formaciones obligatorias para el personal docente y los cargos de responsabilidad administrativos no se han considerado prioritaria por falta de voluntad política.

Además, los recursos económicos destinados a las unidades u observatorios de igualdad y a las otras unidades responsables de estos planes, son en general escasos (Elizondo Lopetegui et al., 2010), hecho que dificulta el desarrollo tanto de actividades de sensibilización como de formación para personal específico y/o de toda la comunidad universitaria. Como consecuencia, frecuentemente las personas encargadas de la atención directa no suelen disponer de una formación específica y se encuentran con dificultades a la hora de dar respuesta a consultas o a peticiones de apoyo y asesoramiento.

En este sentido, consideramos que el problema principal del que adolecen las políticas universitarias para hacer frente a las violencias sexuales y de género es la falta de recursos humanos y económicos que se destinan a la implementación de los protocolos y la ausencia de dialogo e interrelaciones de estos últimos con otras políticas universitarias, tal y como quedó expuesto a lo largo del X Encuentro de Unidades de Igualdad 
de las Universidades Españolas, celebrado en Córdoba en junio 2017 (Unidad de Igualdad de Género UMA, 2017) ${ }^{8}$.

Con todo lo explicado hasta ahora, podemos concluir que, si bien los documentos analizados son presentados como una herramienta disuasoria y al mismo tiempo preventiva y de respuesta ante posibles casos de abusos o violencias (sexuales o de género), en la práctica, tal y como evidencian las entrevistadas, constituyen sobretodo documentos ejecutivos que, si bien tienen por objetivo intervenir y dar respuestas a situaciones traumáticas, son escasamente efectivos a la hora de promover el cambio cultural institucional y colectivo que sería deseable en las políticas universitarias para hacerlas más sensibles a la perspectiva de género. En el próximo apartado analizamos las posibilidades y limitaciones de intervención de estos protocolos frente a casos específicos.

Activando y actuando, que son gerundios

Si consideramos los protocolos como documentos ejecutivos, es sumamente importante fijarnos en quién puede activarlos, ante qué situaciones y cómo, y a quién se tiene que elevar la queja/denuncia, a fin de comprobar hasta qué punto estos protocolos son útiles para dar respuesta a toda la comunidad universitaria o bien terminan por reproducir discriminaciones en su interior.

TABLA 6.

PERSONAS QUE PUEDEN ACTIVAR EL PROTOCOLO 9

\begin{tabular}{lllll}
\hline \multicolumn{1}{c}{ Universidad } & $\begin{array}{c}\text { PDI/ } \\
\text { PAS }\end{array}$ & Estudiantes & $\begin{array}{c}\text { Personal } \\
\text { externo }\end{array}$ & $\begin{array}{c}\text { Otras } \\
\text { usuarias }\end{array}$ \\
\hline UAB, UdG & & & \\
\hline UB, UPC-L, UPC-E, UPF-E, & & & \\
UPF-L, UVIC- UCC & & & \\
\hline UdL, URV & & & \\
\hline UOC & & & \\
\hline
\end{tabular}

Fuente: elaboración propia.

8. Por ejemplo, la segmentación normativa que se produce entre los planes de igualdad y los protocolos impide el desarrollo de respuestas integrales y coordinadas que tengan en cuenta las necesidades de las supervivientes y se planteen como prácticas proactivas y no únicamente reactivas.

9. Nos referimos a lo que en las dimensiones de la tabla 2 se denomina «ámbito de aplicación personal».

10. La protección que se da a las estudiantes en la URV es parcial porque el protocolo se puede activar solo si está involucrada una persona empleada. 
Como se muestra en la tabla 6, los protocolos de la URV y de la UOC son los únicos que excluyen las situaciones de violencia cuando estas se desarrollan entre estudiantes, si bien, en el caso de la URV puede activarse si hay, por lo menos, una persona contratada implicada en la situación de violencia. La UdL sí que prevé poder activar el protocolo en caso de violencias entre estudiantes. En los otros casos analizados, queda también incluido el personal externo que trabaja en las universidades. En los casos de la UAB y de la UdG, estos amplían aún más su espectro: en la UAB al incluir los casos en que estén involucradas usuarias puntuales de servicios/actividades de la Universidad; y en la UdG «en los casos de personas no relacionadas contractualmente o que sean invitadas a participar en algún acto de la Universidad, se activará igualmente el protocolo a los efectos relacionados con la protección de la víctima» (Protocolo de la UdG, p. 5).

En la mayoría de protocolos analizados (UPC-E/UPC-L, UPF-E/UPF-L, UB, UOC, UdG, URV) se otorga la posibilidad de presentar una queja o denuncia por parte de terceros. Los órganos competentes de recibir estas reclamaciones son, en la mayoría de protocolos (URV, UVIC-UCC, UPC-E/UPC-L, UOC, UAB, UPF-L), contadas personas de referencia seleccionadas entre el PAS o el PDI. En opinión de nuestras entrevistadas, su rol académico y la distancia en relación con la mayoría de la comunidad universitaria podrían dificultar la presentación de quejas o denuncias, en particular de las estudiantes. Aún es más complejo cuando estos nombramientos se efectúan de forma discrecional y/o sin considerar los conocimientos y las competencias específicas en atención a supervivientes y la inclusión de la perspectiva de género, ya que la comunidad universitaria puede llegar a identificar estas figuras como representantes de la autoridad en lugar que verlas como consejeras neutrales.

En otros casos, son las unidades/observatorios/comisiones de igualdad (UB, UdL, UdG, UOC, UPF-E) aquellas que se encargan de recabar la denuncia. Aunque este procedimiento parece más coherente con las pautas que debe seguir una atención cuidadosa con las supervivientes, con frecuencia estas unidades no disponen de personal especializado en la atención directa y no se ofrece una supervisión a quienes están encargadas de llevar a cabo esta tarea, lo que conlleva que se centralizen las respuestas, lo que dificulta el acceso y crea malestar en las personas que han de asumir estas tareas sin la consiguiente preparación ni el necesario respaldo.

En general, incluso en las definiciones de las personas y unidades encargadas de acoger las quejas, no se prevé un cuidado específico hacia la persona que sufre. Consideramos que sería necesario incorporar la figura de las «agentes de proximidad», incluyendo a los estudiantes, como figuras cercanas y fácilmente asequibles, capaces de detectar y responder a estas situaciones, y de acompañar a las personas en su proceso.

En algunos casos, además, se evidencia la distancia entre lo enunciado por el documento y aquello que se aconseja hacer en la práctica diaria. Por ejemplo, en el protocolo de la UAB se especifica que para presentar la denuncia se tiene que enviar en un sobre cerrado a través del Registro General a la atención del Rectorado, cuando en la práctica es el Observatorio de Igualdad quien se encarga de la acogida y asesoramiento a las personas que lo necesitan. Este cambio, implementado a partir de decisiones políticas para la mejora de la intervención, no ha quedado de momento registrado en el 
documento. Esto se debe a que estas normativas, con frecuencia, son el resultado de profundas y largas negociaciones que, como nos comentaba una de las expertas entrevistadas, pueden incluir "cambios de gobierno a mitad del proceso". En opinión de muchas de las expertas, esta circunstancia ha provocado que con demasiada frecuencia sus aprobaciones y modificaciones sean complejas y agotadoras. A su vez, la rigidez documental repercute en la poca adaptabilidad de estos documentos para dar respuesta a situaciones específicas e incorporar los efectos de la interseccionalidad en las vivencias de los abusos y las violencias.

Otro elemento que debe tenerse en cuenta son los espacios en los cuales la Universidad quiere asumir responsabilidades. Algunos protocolos (excluyendo los de la URV, la UB y la UOC) se pueden activar también en determinados espacios externos a la universidad siempre y cuando se desarrolle una actividad académica, laboral o profesional. En el caso de la UPC-E se contempla también su activación cuando la Universidad es responsable de la organización de otras actividades (deportivas, festivas, etc.) y en aquellos casos en que los y las estudiantes realizan prácticas en empresas externas. En el caso de la UdL se puede activar cuando «aprovechando la relación especial de sujeción que pueda generar la actividad laboral o educativa entre la persona transgresora y la persona ofendida, asediada o agredida, al margen del lugar donde se hayan acontecido estos comportamientos» (p. 9). Mientras, los de la UPF-E/UPF-L y la UOC permiten iniciarlos en casos de violencia a través de internet y en las redes sociales. Por último, la UdG reconoce la posibilidad de activar su protocolo si los hechos tienen lugar fuera de sus instalaciones y están relacionados con los desplazamientos (de acceso o salida).

\section{Suma y sigue}

Aun analizando estos documentos y teniendo en cuenta que se centran en la intervención ante casos concretos, detectamos que una de las causas de la ineficiencia de dichas políticas es la ausencia de medidas cautelares - en algunos casos ni son nombradas (UAB, UPC-L) —. Tal y como evidencian la mayoría de las personas entrevistadas, cuando se activan estas disposiciones normalmente son las personas acosadas la que van a sufrir repercusiones ulteriores en su vida cotidiana. Por ejemplo, al ser separadas del resto de su clase para hacer un examen otro día u «ofreciéndoles» el traslado a otra oficina o centro. Quizás por esta razón, el 9 de mayo de 2017 las juntas y los comités de los trabajadores de la URV (tanto PDI como PAS) emitieron un comunicado en el cual evidenciaban cómo la falta de medidas cautelares ha comportado perjuicios para quienes realizaron una denuncia en 2014. El mal funcionamiento de tales medidas - que genera una victimización secundaria y que puede provocar nuevos daños o perjuicios psicológicos, sociales o económicos a las personas acosadas (Beristain, 1996) - es particularmente perjudicial cuando la persona decide activar vías externas y denunciar penalmente los hechos, ya que en estos casos los procedimientos internos se bloquean hasta la resolución judicial, dejando desamparada a la persona 
denunciante. Lo mismo sucede con las medidas correctivas y sancionadoras, escasamente detalladas en casi todos los documentos analizados.

Paradójicamente, en los protocolos se enfatiza el hecho que las quejas o las denuncias puedan ser falsas (URV, UB, UdL, UPF 2016, UdG). Por ejemplo, en el protocolo de la UdG se menciona la posibilidad de "denuncias falsas que se hayan presentado en mala fe" (p. 14), y en el de la UPF 2016 se explicita que hay que dejar claras las consecuencias en caso de denuncia (p. 9). Sin embargo, estas prevenciones contrastan con la realidad, ya que, de acuerdo con la Fiscalía del Estado (2016), las cifras de denuncias falsas en caso de violencias de género - la otra área en la que continuamente entra esta retórica en los debates - son especialmente bajas en relación con otros tipos de delito.

Estamos así frente a un mito (Bodelón, 2014) o un neomito (Lorente, 2009) que sirve para minimizar, naturalizar o negar las mismas violencias. El hecho de que esta posibilidad aparezca siempre mencionada en dichos documentos universitarios constituye, en nuestra opinión, una demostración de las resistencias a reconocer directamente el problema por parte de la institución y, probablemente, también sean el resultado de las negociaciones desarrolladas durante su elaboración entre personas que tienen diferentes sensibilidades al respecto. Esta idea se refuerza si consideramos que algunos documentos tampoco reconocen el riesgo en que incurren las personas que denuncian sufrir represalias (URV, UdG, UAB), aunque la mayoría de universidades explican claramente que no se aceptarán estos tipos de conductas, sin detallar medidas específicas para el caso. Un caso excepcional es el de la UdL, que en su anexo 1 reconoce la "no indemnidad" en los casos de represalias como una forma de discriminación por razón de género, punible según estas características.

Esta postura de no responsabilizarse de la protección de la persona superviviente se alinea perfectamente con una cultura institucional y organizativa de las instituciones académicas (Handy, 1993) conservadora y defensora del statu quo, y a una visión no compartida sobre sus obligaciones y responsabilidades frente a los posibles casos de violencias sexuales en su comunidad. Así, el simple hecho de disponer de políticas y procedimientos no parece suficiente para determinar el compromiso efectivo de las instituciones de enseñanza superior a la hora de abordar este problema.

En resumen, consideramos que los protocolos constituyen disposiciones necesarias, pero no suficientes para luchar contra las violencias sexuales en los contextos institucionales analizados. En primer lugar, porque ponen demasiado énfasis en las medidas sancionadoras y procedimentales sin contemplar, simultáneamente, la necesidad de cuidado, apoyo y atención que necesitan las supervivientes; carencia que podría cubrirse a través de una mayor formación/sensibilización de la comunidad universitaria y con la creación de servicios específicos. En segundo lugar, porque no tienen en cuenta de manera explícita cómo intervienen las jerarquías ni las relaciones de poder generizadas presentes en la institución. Omitir esta realidad implica acentuarla y perpetuarla en el tiempo y en el espacio con el propósito de mantener el orden establecido (Cantillo y Rocha, 2007). Esta omisión tiene como consecuencia que no se realice un abordaje integral del problema en tanto que se invisibilizan sus raíces. 
Finalmente, el no reconocimiento del poder de agencia a las personas agredidas y acosadas, que no pueden intervenir en las decisiones sobre los pasos que se deben realizar una vez activado el protocolo, juntamente con las malas e inadecuadas prácticas que pueden llegar a producirse, pueden generar ulteriormente situaciones de victimización secundaria que pueden convertirse, a su vez, en otra forma de violencia en los casos en que aquellas acciones $\mathrm{u}$ omisiones de las universidades provoquen nuevos casos de violencias, así como por parte de aquellas acciones que muestren una discriminación u obstaculización para que las personas acosadas puedan ejercer sus derechos (Bodelón, 2014). Estos elementos, en un contexto cargado de mitos y estereotipos alrededor de las violencias sexuales y de la sexualidad generizada, tienden a enmascarar las experiencias de abusos y violencias, e implican que muchas veces estos procesos conlleven la descalificación, el descrédito, la falta de empatía, así como la imposición de ritmos y/o actuaciones que favorecen nuevamente la revictimización de las supervivientes (Calle, 2004).

\section{CONCLUSIONES: Y AHORA... ¿QUÉ?}

Las violencias sexuales en las universidades catalanas son un problema todavía bastante desconocido y subestimado, al cual no se le han dado las respuestas necesarias. Reconocer la existencia de este fenómeno más allá de las declaraciones institucionales y comprender y enfrentar la influencia de la relación de poder en la definición de las violencias sexuales constituyen tareas indispensables e impostergables.

Por una parte, el análisis realizado evidencia que los protocolos aprobados por las universidades catalanas para este fin surgen en parte para adaptarse al nuevo marco legal, pero muestran escasa convicción en la incorporación de la perspectiva de género. De hecho, se evidencia que las peticiones de los movimientos feministas han sido cooptadas por las instituciones a través de la aprobación de políticas poco efectivas y procedimientos muy rígidos que terminan por neutralizarlas, además de no adaptarse a la gran variedad de situaciones presentes hoy en día en nuestras universidades.

Por otra parte, la cada vez mayor internacionalización de la enseñanza superior, la privatización de determinados servicios y el crecimiento de contratos de trabajo temporales y precarios que caracterizan a las universidades del siglo XXI, las convierten en instituciones cada vez más complejas, cuyas respuestas frente a las violencias de género y sexuales que se desarrollan en su interior tendrían que ser más integrales y funcionales (Paz et al, 2016).

En nuestra opinión, los protocolos actualmente en uso corren el riesgo de convertirse en instrumentos que permitan a los órganos de gobierno no asumir su responsabilidad en la cuestión. De este modo, su mera existencia permitiría a las autoridades académicas escudarse frente a las críticas feministas, al declarar haber cumplido con las obligaciones legales, pero sin tomar en consideración el alcance y eficacia real de tales medidas. De la misma manera, la escasa aplicación de los procedimientos universitarios en los casos de violencias y de acoso, lejos de ser interpretada como una limitación de los mismos, les permitiría mantenerse en la inopia ante la situación. Tal y como 
sugieren las distintas entrevistas, sigue existiendo una reticencia institucional a reconocer la existencia del fenómeno porque se considera que dicho reconocimiento podría dañar la imagen y la reputación de la institución universitaria.

Una revisión de estos procedimientos implica la necesidad de actualizar la legislación sobre la cual se fundamentan los protocolos y los mecanismos de investigación y sanción de los que disponen las universidades. Además, es necesaria una reforma que vaya más allá del Pacto de Estado sobre Violencia de Género, ya que es imprescindible que el Estado asuma el deber de prevenir y atender el conjunto de violencias sexuales y de género, no solo aquellas que se producen en el ámbito de la pareja, sino también en el espacio público, las aulas, los entornos profesionales y, en general, en todos los espacios públicos y privados.

Sin embargo, tal y como hemos podido comprobar a lo largo del proyecto USVReact, consideramos que no es posible abordar fenómenos complejos, como son las violencias sexuales y de género, partiendo únicamente de un punto de vista normativo, y aún menos a partir de acciones simplemente de control y/o punitivas. De hecho, distintas especialistas llevan ańos criticando que «la demanda del movimiento de mujeres para criminalizar la violencia sexual ha sido satisfecha de forma inadecuada, puesto que se ha reducido el significado que para el movimiento de mujeres tenía hablar de violencia sexual. Es decir, la criminalización del problema restringe su significado» (Bergalli y Bodelón, 1992: 64).

Por tanto, consideramos que los protocolos no pueden y no deben ser las únicas políticas presentes en el entorno universitario. Es necesario y urgente desarrollar políticas más amplias en las que la Universidad asuma su responsabilidad de manera clara, y que proporcione aquellos recursos necesarios (humanos, materiales y económicos) para su adecuada implementación. En este sentido, consideramos que las medidas preventivas y formativas no pueden seguir basándose en una exposición de buenas intenciones escritas en un documento que nadie conoce, por lo que es necesario hacer una divulgación completa del protocolo a través de todas las herramientas disponibles (carpetas estudiantiles, espacios docentes, baños, páginas web, intranet universitaria, etc.) y fortalecer aquellos aspectos de prevención y formación que más fielmente cumplen la misión otorgada a las universidades. Para ello, todas deberíamos implicarnos en la creación de una red social y universitaria que promueva actividades que subviertan la cultura de la violación (Phipps et al, 2018) y promuevan un ambiente y enfoque respetuoso con los problemas que enfrenta la persona superviviente. En este sentido, las universidades del siglo Xxi tienen una doble responsabilidad: la de erradicar determinadas prácticas violentas y discriminatorias en sus espacios y la de colaborar en la formación de nuevas generaciones de profesionales libres de prejuicio y capaces de intervenir en la creación de una sociedad más equitativa y sin violencia.

\section{AGRADECIMIENTOS}

Esta nota de investigación está enmarcada en la investigación-acción USVReact (JUST/2014/RDAP/AG/VICT/7401), coordinada por Pam Aldred, de la Brunel University y, en el contexto catalán, por Barbara Biglia. El análisis del contexto legal 
es también deudor del trabajo realizado en el marco de otro proyecto, el GAPwork (JUST/2012/DAP/AG//3176), bajo la misma coordinación, en el que también colaboró María Olivella. Ambos proyectos han sido cofinanciados por la Comisión Europea. Sin embargo, las publicaciones y comunicaciones que derivan de ellos reflejan únicamente las visiones de sus autoras, y la Comisión no se hace responsable de cualquier uso derivado de las informaciones contenidas en las mismas.

Agradecemos, en primer lugar, todas las participaciones en los dos proyectos: las personas formadas, las investigadoras, las formadoras y los comités científicos de suporte. En segundo lugar, queremos expresar nuestro agradecimiento al Jordi Bonet-Martí, por su apoyo y sugerencias, a Eloísa Pińeiro Orge por la cuidadosa edición del castellano, así como a las evaluadoras anónimas por los comentarios a la primera versión de este texto.

\section{Referencias}

Alldred, Pam y Barbara Biglia. 2015. «Gender-Related Violence and Young People: An Overview of Italian, Irish, Spanish, UK and EU Legislation», Children and Society, 29 (6): 662-675. Disponible en: https://doi.org/10.1111/chso.12141.

Amorós, Celia. 2008. "Conceptualizar es politizar», en Patricia Laurenzo Copello, María Luisa Maqueda Abreu y Ana María Rubio Castro (coords.), Género, violencia y derecho. Valencia: Tirant lo Blanch.

Ballarín Domingo, Pilar. 2015. «Los códigos de género en la Universidad», Revista Iberoamericana de Educación, 68: 19-38. Disponible en: https://doi.org/10.35362/ rie680168.

Bardina Martín, Yolanda y María Murillo Quintilla. 2013. Percepción de la violencia de género en el entorno universitario. El caso del alumnado de Lleida. Lleida: Universidad de Lleida.

Bergalli, Roberto y Encarna Bodelón. 1992. «La cuestión de las mujeres y el derecho penal simbólico", Anuario de Filosofía del Derecho, 9: 43-74.

Beristain, Antonio. 1996. Criminología, victimología y cárceles. Bogotá: Javegraf.

Biglia, Barbara. 2015. «Aclarando términos: el paradigma de las violencias de género", en Barbara Biglia y Edurne Jímenez (coords.), Jóvenes, género y violencias: hagamos nuestra la prevención. Tarragona: Universitat Rovira i Virgili. Disponible en: https://doi.org/10.17345/9788484243571.

Biglia, Barbara y Edurne Jiménez. 2018. «Respuestas formativas radicales para afrontar un problema estructural y capilar», en Beatriz Santamarina (coord.), Mujeres y Universidad (sin cifras). La violencia invisible. Alzira: Neopàtria.

Biglia, Barbara y Jordi Bonet i Martí. 2017. «DIY: Towards feminist methodological practices in social research", Annual Review of Critical Psychology, 13: 1-16.

Biglia, Barbara y Nuria Vergé. 2016. «Qüestionant la perspectiva de gènere en la recerca», Revista d'Innovació i Recerca en Educació (REIRE), 9 (20): 12-29.

Biglia, Barbara y Sara Cagliero. En prensa. «Abordajes y "respuestas" de las universidades catalanas frente a las violencias LGTBIQ+fóbicas». Quaderns de Psicologia. 
Bodelón, Encarna. 2014. "Violencia institucional y violencia de género», Anales de la Cátedra Francisco Suárez, 48: 131-155.

Bodelón, Encarna, Nuria Igareda y Gloria Casas. 2012. Violencia sexual, acoso y miedo al delito: informe español. Bellaterra: Universitat Autonoma de Barcelona.

Bonet, Jordi. 2007. «Problematizar las políticas sociales frente a la(s) violencia(s) de género", en Barbara Biglia y Concha San Martín (coords.), Estado de Wonderbra. Entretejiendo narraciones feministas sobre las violencias de género. Barcelona: Virus.

Borraz, Marta. 2017. «La ley no reconoce el juicio a La Manada por violación como un proceso por violencia machista», Eldiario.es. 29-11-2017. Disponible en: http://bit.ly/2NDMLHt.

Bosch, Esperanza y Victoria Ferrer. 2000. «La violencia de género: de cuestión privada a problema social», Psychosocial Intervention, 9 (1): 7-19.

Bosch, Esperanza, Victoria Ferrer y Aina Alzamora. 2006. El laberinto patriarcal. Barcelona: Antrophos.

Bustelo, Maria. 2014. «Evaluación de políticas públicas con perspectiva de género», en Marta Lois y Alba Alonso (coords.), Ciencias politicas con perspectivas de género. Madrid: Akal.

Bustelo, María y Emanuela Lombardo. 2007. «¿Qué hay debajo de la alfombra de las políticas de igualdad? Un análisis de marcos interpretativos en España y en Europa», en María Bustelo y Emanuela Lombardo (eds.), Politicas de igualdad en España y en Europa. Valencia: Cátedra.

Bustelo, María y Emanuela Lombardo. 2012. "Understanding and assessing quality in gender violence policies in Italy and Spain», en Thinking Big about "Gender Equality" policy in the comparatie politics of gender. Antwerp: ECRP joint sessions.

Cagliero Sara y Barbara Biglia. 2016. «Critica femminista alle norme italiane sulle violenze di genere», AG About Gender-Rivista internazionale di studi di genere, 5 (10).

Calle, Soledad. 2004. Consideraciones sobre la victimización secundaria en la atención social a las víctimas de la violencia de género. Hospitalet de Llobregat: Ayuntamiento de l'Hospitalet de Llobregat.

Cantillo, Ligia y Delma Rocha. 2007. «Las relaciones de género y la educación superior en Colombia», en Rafaela Vos Obeso (coord.), Sociología e historia: un diálogo interdisciplinario con el feminismo y el género. Puerto Colombia: Universidad del Atlántico.

Cela, Daniel. 2017. «El exdecano condenado por abusar de tres profesoras dice que los tocamientos fueron "consentidos"», Público.es, 1-5-2017. Disponible en: http:// bit.ly/32en5UR.

Chuaqui J., Benedicto. 2002. "Acerca de la historia de las universidades», Revista Chilena de Pediatría, 73 (6): 583-585. Disponible en: http://bit.ly/329ZDZ7.

Elizondo Lopetegui, Arantxa, Ainhoa Novo Arbona y María Silvestre Cabrera. 2010. Igualdad de mujeres y hombres en las universidades españolas. Madrid: Instituto de la Mujer. Disponible en: http://bit.ly/2JmwrXr. 
Europa Press. 2017. «Siete años de cárcel a un catedrático de la Universidad de Sevilla por abuso sexual a tres profesoras", Eldiario.es, 9-1-2017. Disponible en: http:// bit.ly/2JzwyOl.

Facio, Alda. 2004. «Metodología para el análisis de género de un proyecto de ley», Otras Miradas, 4 (1): 1-11.

Feltes, Thomas, Augusto Balloni, Janina Czapska, Encarna Bodelón y Philip Stenning. 2012. Gender-based violence, stalking and fear of crime. Research project 20092011 Research Report. Bochum: Ruhr-Universität Bochum. Disponible en: http:// bit.ly/2NAtlTB.

Fernández Díaz, Natalia. 2003. La violencia sexual y su representación en la prensa. Barcelona: Anthropos.

Fiscalía General del Estado. 2016. Memoria. Madrid: Centro de Estudios Jurídicos. Ministerio de Justicia. Disponible en: http://bit.ly/2Jl26IB.

Gil Ruiz, Juana María. 2012. El convenio de Estambul. Como marco de derecho antisubordiscriminatorio. Madrid: Dykinson.

Gross, Alan M., Andrea Winslett, Miguel Roberts y Carol L. Gohn. 2006. «An examination of sexual violence against college women", Violence Against Women, 12: 288-300. Disponible en: https://doi.org/10.1177/1077801205277358.

Handy, Charles. 1993. Understanding organizations. Londres: Penguin Books.

Igareda, Noelia y Marta Cruells. 2014. «Críticas al derecho y el sujeto «mujeres» y propuestas desde la jurisprudencia feminista», Cuadernos Electrónicos de Filosofía del Derecho, 30, 1-11.

Jimenez, Edurne, Barbara Biglia y Sara Cagliero. 2016. «Apunts per millorar les formacions sobre violències de gènere. L'Experiecia de la investigació-acciò feminista GAPWork», Pedagogia i Treball Social. Revista de Ciències Socials Aplicades, 5 (1): 79-104.

Larrauri, Elena. 2007. Criminología critica y violencia de género. Madrid: Trotta.

Lorente, Miguel. 2009. Los nuevos hombres nuevos. Barcelona: Destino.

Luxán, Marta, Barbara Biglia y Jokin Azpiazu (eds.). 2018. Violencias sexuales: una asignatura pendiente. Guia para afrontar las violencias sexuales en las universidades. Leioa: UPV/EHU.

Marshall, Rachel. 2014. «Will it really save you? Analyzing the campus sexual violence elimination act», Legislation and Policy Brief, 6: 271-293.

Palermo, Alicia. 2006. «El acceso de las mujeres a la educación universitaria», Revista Argentina de Sociologia, 4 (7): 11-46. Disponible en: http://bit.ly/32hWZ3l.

Paz Enrique, Luis Ernesto, Eduardo Alejandro Hernández, Alfonso y Hans Van de Water. 2016. «Los retos de la Educación Superior en el Siglo XxI», Revista Conrado, 12 (55): 17-24.

Phipps, Alison, Jessica Ringrose, Emma Renold y Carolyn Jackson. 2018. «Rape culture, lad culture and everyday sexism: researching, conceptualizing and politicizing new mediations of gender and sexual violence», Journal of Gender Studies, 27: 1-8. Disponible en: https://doi.org/10.1080/09589236.2016.1266792. 
Phipps, Alison y Geraldine Smith. 2012. «Violence against women students in the UK: time to take action", Gender and Education, 24 (4): 357-373. Disponible en: https://doi.org/10.1080/09540253.2011.628928.

Plataforma CEDAW Sombra España. 2017. Informe sobre seguimiento parcial de las observaciones finales del Comité CEDAW en el examen del Estado Español de julio 2015 (CEDAW/C/ESP/7-8). Disponible en: http://bit.ly/2LJlpgz.

Salazar, Óscar. 2018. «Paridad y justicia de género. Algunas reflexiones críticas sobre las políticas de igualdad en España», Revista Comunicación y Género, 1 (1): 5-24. Disponible en: https://doi.org/10.5209/CGEN.60245.

Segato, Rita. 2003. Las estructuras elementales de la violencia. Buenos Aires: Prometeo.

Toledo, Patsilí, Encarna Bodelón González, Neus Tur Bujos y M. Jimena Martínez Berruete. 2016. Marc juridic internacional, estatal i autonòmic de les violències sexuals (matrimonis forcats, mutilacions genitals femenines, tràfic d'éssers humans amb finalitat d'explotació sexual, assetjament $i$ agressions sexuals). Bellaterra: Universitat Autònoma de Barcelona, Grup de Recerca Antígona.

Toledo, Patsilí y Montse Pineda. 2016. L'abordatge de les violències sexuals a Catalunya. Barcelona: Bellaterra.

Unidad de Igualdad de Género UMA. 2017. Conclusiones finales del X Encuentro de Unidades de Igualdad Españolas. Disponible en: http://bit.ly/2xyS7ZL.

Velázquez, Susana. 2003. Violencias cotidianas, violencia de género. Escuchar, comprender, ayudar. Buenos Aires: Paidós.

Verge, Tània y Teresa Cabruja. 2017. La perspectiva de gènere en docència i recerca a les universitats de la Xarxa Vives. Situació actual i reptes de futur. Castelló de la Plana: Xarxa Vives.

Referencia de leyes citadas

Ley Orgánica 10/1995, de 23 de noviembre, del Código Penal. Boletín Oficial del Estado, 281, 24-11-1995. Arts. 178-194. Disponible en: http://bit.ly/2Lv1zGi.

Ley Orgánica 1/2004, de 28 de diciembre, de Medidas de Protección Integral contra la Violencia de Género. Boletin Oficial del Estado, 313, 29-12-2004. Disponible en: http://bit.ly/30KViKr.

Ley Orgánica 2/2006, de 3 de mayo, de Educación. Boletín Oficial del Estado, 106, 4-5-2006. Disponible en: http://bit.ly/2XXhv6d.

Ley Orgánica 3/2007 para la Igualdad Efectiva entre Mujeres y Hombres. Boletín Oficial del Estado, 71, 23-3-2007. Disponible en: http://bit.ly/2Z15e1E.

Ley Orgánica 4/2007, de 12 de abril, por la que se modifica la Ley Orgánica 6/2001, de 21 de diciembre, de Universidades. Boletin Oficial del Estado, 89, 13-4-2007, págs. 16241-16260. Disponible en: http://bit.ly/2YZwTjB.

Ley $5 / 2008$, de 24 de abril, del derecho de las mujeres a erradicar la violencia machista del Parlament de Catalunya. Butlleti Oficial del Parlament de Catalunya, 251/VIII, 28-4-2008. Disponible en: http://bit.ly/2M2ffry. 
Ley 11/2014, de 10 de octubre, para garantizar los derechos de lesbianas, gays, bisexuales, transgéneros e intersexuales y para erradicar la homofobia, la bifobia y la transfobia, Comunidad Autónoma de Cataluña. Boletín Oficial del Estado, 281, 20-11-2014. Disponible en: http://bit.ly/2xSjFcG.

Presentado para evaluación: 25 de junio de 2018.

Aceptado para publicación: 26 de junio de 2019.

SARA CAGLIERO
sara.cagliero@gmail.com

Licenciada en Ciencias Políticas por la Universidad de Turín (Italia), con máster en Estudios de Mujeres, Género y Ciudadanía por el Instituto Interuniversitario de Mujeres y Género (IIEDG). Actualmente es doctoranda en Humanidades con especialización en Estudios de Género en la Universitat Rovira i Virgili (URV), técnica de proyectos en el grupo GREDI-URV y miembro del Seminario Interdisciplinar de Metodología de Investigación Feminista (SIMREF). Ha obtenido numerosas becas en instituciones nacionales e internacionales, como la Comisión Europea, y actualmente participa en diferentes proyectos de investigación, tanto a nivel nacional como internacional. Buena parte de su producción académica puede consultarse en https://urv. academia.edu/SaraCagliero.

\section{BARBARA BIGLIA}

barbara.biglia@urv.cat

Doctora en Psicología por la Universidad de Barcelona, con mención de doctorado europeo. Actualmente, es profesora agregada Serra Hunter en el Departamento de Pedagogía de la URV. Investiga bajo el prisma de la perspectiva de género y feminismo, tratando temáticas diversas como los procesos de producción de conocimiento y la epistemología feminista, las violencias de género, el análisis sociológico de políticas públicas, etc. Es fundadora y miembro activo del SIMREF desde 2008 y es también miembro del grupo de investigación consolidado interuniversitario GREDI-URV, así como del IIEDG. Ha sido coordinadora catalana de los proyectos EU GAPwork y USVReact y es actualmente investigadora principal del proyecto "Visibilizar y dimensionar el problema de las violencias sexuales y de género en las universidades". Buena parte de su producción académica puede consultarse en https://urv.academia.edu/ BarbaraBiglia. 